\title{
LHC: Standard Higgs and Hidden Higgs
}

\author{
Christoph Englert, ${ }^{1}$ Tilman Plehn, ${ }^{2}$ Michael Rauch, ${ }^{3}$ Dirk Zerwas, ${ }^{4}$ and Peter M. Zerwas ${ }^{5,6}$ \\ ${ }^{1}$ Institute for Particle Physics Phenomenology, Department of Physics, Durham University, United Kingdom \\ ${ }^{2}$ Institut für Theoretische Physik, Universität Heidelberg, Germany \\ ${ }^{3}$ Institut für Theoretische Physik, Karlsruhe Institute of Technology, Germany \\ ${ }^{4} L A L$, IN2P3/CNRS, Orsay, France \\ ${ }^{5}$ Deutsches Elektronen-Synchrotron DESY, Hamburg, Germany \\ ${ }^{6}$ Institut für Theoretische Teilchenphysik und Kosmologie, RWTH Aachen University, Germany
}

(Dated: March 14, 2012)

\begin{abstract}
Interpretations of Higgs searches critically involve production cross sections and decay probabilities for different analysis channels. Mixing effects can reduce production rates, while invisible decays can reduce decay probabilities. Both effects may transparently be quantified in Higgs systems where a visible Higgs boson is mixed with a hidden sector Higgs boson. Recent experimental exclusion bounds can be re-interpreted in this context as a sign for non-standard Higgs properties. Should a light Higgs boson be discovered, then our analysis will quantify how closely it may coincide with the Standard Model.
\end{abstract}

\section{BASICS}

Recent experimental searches for Higgs bosons [1] by ATLAS [2] and CMS [3], based on luminosities of up to about $2.3 \mathrm{fb}^{-1}$, have excluded the Higgs boson of the Standard Model, when combined [4], between $141 \mathrm{GeV}$ and $476 \mathrm{GeV}$ at 95\% CL. ATLAS and CMS have updated several channels using an integrated luminosity of up to $5 \mathrm{fb}^{-1}[5,6]$, so that each experiment alone has about the same sensitivity as the combination in the low mass region, raising the low-mass bound of the Higgs from the LEP2 limit [7] of $114.4 \mathrm{GeV}$ to $115.5 / 115 \mathrm{GeV}$ and leading to an exclusion of the mass from $131 \mathrm{GeV} / 127 \mathrm{GeV}$ to $453 \mathrm{GeV} / 600 \mathrm{GeV}$ by ATLAS/CMS, respectively. Bounds as low as fractions $\mathcal{R} \leq 0.3$ have been set on the production cross sections with respect to the Standard Model [SM] for some of the masses probed. Given the far reaching consequences, the question arises naturally to what extent Higgs bosons, in slightly generalized scenarios, may still exist in this mass region.

On the other hand, a gap from about $130 \mathrm{GeV}$ down to the low-mass limit of about $115 \mathrm{GeV}$ is left, presently, in which a SM-type Higgs signal can be expected to either rise up or plunge in the near future [5, 6]. When a Higgs boson will indeed be discovered in this mass range, the standard-hidden Higgs scenario allows to quantify how well the global properties of the particle coincide with the predictions of the Standard Model.

Both these questions have been addressed [8,9] at the theoretical level in a standard-hidden Higgs scenario. The present sequel will include the recent LHC results on the Higgs sector in the analysis. The ground for the fundamental idea to use the Higgs boson as a portal to a novel hidden sector in nature has been laid for general model structures in Ref. [11]. Phenomenological implications [12] can then be studied in specific frameworks like hidden valleys [13]. Other than the Higgs portal there exist two additional portal-type interactions in a renormalizable theory, namely kinetic $U(1)$ mixing [14] and mixing with sterile neutrinos [15].

The experimentally observed rates depend on the Higgs production cross sections and the decay branching ratios [16]. In scenarios in which the standard Higgs boson is mixed with another Higgs boson, originating from a hidden sector for instance, the production cross sections are reduced universally by the mixing parameter and, in addition, the visible branching ratios may be lowered by decays into invisible channels induced by mixing. Throughout this paper we will consider the minimal Standard Model Higgs sector as the activator of the visible Higgs boson [17], but the same arguments trivially hold for extended visible Higgs sectors, for example in supersymmetry or other extensions to the Standard Model [18]. In the early theoretical analyses of Refs. [8,9] the two effects have been joined properly, expanded here by exploiting data in addition.

Specific examples have also been discussed in Ref. [19]. The opening of invisible channels has been investigated in several other studies, motivated by a large variety of models ranging from Higgs decays to LSP's in supersymmetry [20] and other SM extensions, see e.g. [21], to phenomenological approaches [22, 23], partly connected with cold dark matter analyses [24]. However, the effect of mixing on the production cross sections has often been left aside.

The Higgs potential of the standard-hidden Higgs system consists of the standard potential in the visible sector and the isomorphic potential in the hidden sector, coupled by a bi-bilinear portal term [11]. Vacuum expectation values of the Higgs fields build up the Higgs mass parameters in the two sectors as well as their coupling. Diagonalizing the standard-hidden Higgs mass matrix [squared] ${ }^{*}$, generates the mass eigenvalues $M_{1}^{2}<M_{2}^{2}$ and rotates the standard-hidden Higgs states $H_{s}, H_{h}$ to the

*The essential elements of the formalism $[8,9]$ are summarized briefly in this introductory section as to render the letter self-contained for the reader's convenience. 
mass eigen-states $H_{1}, H_{2}$,

$$
\begin{aligned}
& H_{1}=\cos \chi H_{s}+\sin \chi H_{h} \\
& H_{2}=-\sin \chi H_{s}+\cos \chi H_{h},
\end{aligned}
$$

the labels ordered parallel to the rising mass values. In the limit of small mixing, $H_{1}$ approaches the lighter of the states $H_{s}$ or $H_{h}$, i.e. $\cos \chi$ or $\sin \chi \rightarrow 1$, respectively, and $H_{2}$ the heavier of the two primary states.

The mixing reduces the production cross sections of $H_{1}, H_{2}$ to

$$
\sigma_{1,2}=\cos ^{2} \chi\left\{\sin ^{2} \chi\right\} \sigma_{1,2}^{\mathrm{SM}}
$$

with respect to the cross sections of the Standard Model for equivalent masses, the expressions within the curly brackets for index $=2$ substituting the corresponding expressions for $i n d e x=1$. The visible and invisible partial widths are modified correspondingly,

$$
\begin{aligned}
& \Gamma_{1,2}^{\mathrm{vis}}=\cos ^{2} \chi\left\{\sin ^{2} \chi\right\} \Gamma_{1,2}^{\mathrm{SM}}+\Delta_{2}^{v i s} \Gamma_{2}^{\mathrm{HH}} \\
& \Gamma_{1,2}^{\mathrm{inv}}=\sin ^{2} \chi\left\{\cos ^{2} \chi\right\} \Gamma_{1,2}^{\mathrm{hid}}+\Delta_{2}^{i n v} \Gamma_{2}^{\mathrm{HH}}
\end{aligned}
$$

are built up by decays to particles in the hidden sector, including cascade decays with $\Delta_{2}^{\text {vis }\{\text { inv }\}}=\zeta_{1}^{2}\left\{\left[1-\zeta_{1}\right]^{2}\right\} \neq 0$ only for $i n d e x=2$. After factorizing off the mixing parameters, the relevant $H_{h}$ widths $\Gamma_{1,2}^{\text {hid }}$, defined for virtual masses $M_{1,2}$, are generated by the unknown dynamics of the hidden sector. Most agnostically, these width parameters should not exceed the values of the Higgs masses, $\Gamma_{1,2}^{\text {hid }} \leq M_{1,2}$. The partial width $\Gamma_{2}^{\mathrm{HH}}$ accounts for potential $H_{2} \rightarrow H_{1} H_{1}$ cascade decays if $M_{2}>2 M_{1}$ [19] with the $H_{1}$ visible decay branching ratio $\zeta_{1}=1 /\left[1+\tan ^{2} \chi \Gamma_{1}^{\text {hid }} / \Gamma_{\text {tot }, 1}^{\mathrm{SM}}\right]$. In this case also a mixed contribution $\Gamma_{2}^{\mathrm{HH}, \mathrm{mix}}=2 \zeta_{1}\left[1-\zeta_{1}\right] \Gamma_{2}^{\mathrm{HH}}$ of one $H_{1}$ decaying visibly and the other one invisibly is present; numerical details have been presented in Ref. [9]. Likewise the total widths,

$$
\Gamma_{1}^{\mathrm{tot}}=\cos ^{2} \chi\left\{\sin ^{2} \chi\right\} \Gamma_{\mathrm{tot} ; 1,2}^{\mathrm{SM}}+\sin ^{2} \chi\left\{\cos ^{2} \chi\right\} \Gamma_{1,2}^{\mathrm{hid}}+\Delta_{2} \Gamma_{2}^{\mathrm{HH}},
$$

with $\Delta_{2}=0,1$ for $i n d e x=1,2$, respectively, are free parameters at the phenomenological level in this scenario.

\section{HIGGS MEASUREMENTS VS HIGGS PARAMETERS}

The search for Higgs bosons at the LHC by ATLAS [2] and CMS [3] has excluded major parts of the intermediate Higgs mass range and beyond [4-6]. However, it leaves a clear gap down to the low-mass limit set originally by LEP2 and improved by ATLAS/CMS to about $115 \mathrm{GeV}$ in which a Higgs signal may be confirmed or refuted in the coming year [5, 6]. In this Section we follow two scenarios. First, we study Higgs sectors with reduced couplings and invisible decay widths, which can naturally explain a missing Higgs signal at the LHC. In a second step, we describe the effects of a potential Higgs discovery in the still open mass window with SM-like couplings.

\section{Bounds}

To begin with, in the Higgs mass range ruled out for Standard Model couplings, the corresponding Higgs cross sections are constrained to values as low as $\mathcal{R} \sim 0.3$ in units of the SM value. These bounds, as well as production cross sections measured after the discovery of the Higgs boson, can be re-interpreted within the standard-hidden Higgs scenario. For a given mass value the bounds induce constraints on the mixing and on the invisible width of $H_{1}$, while at the same time the second, heavier state of the Higgs pair is proven not to violate the experimental bounds. The bounds on the production cross sections for $H_{1}$ from any set of final states $F$ induce the following constraints at a given mass value on the mixing parameter and the invisible width:

$$
\frac{\sigma\left[p p \rightarrow H_{1} \rightarrow F\right]}{\sigma\left[p p \rightarrow H_{1} \rightarrow F\right]^{\mathrm{SM}}}=\frac{\cos ^{2} \chi}{1+\tan ^{2} \chi\left[\Gamma_{1}^{\mathrm{hid}} / \Gamma_{\mathrm{tot}, 1}^{\mathrm{SM}}\right]} \leq \mathcal{R}
$$

[This observable coincides with the twin ratio $\Gamma_{\mathrm{p}} \Gamma_{\mathrm{d}} / \Gamma_{\text {tot }}$ of Refs. [8, 9], composed of partial widths for the production and decay channels as well as the total decay width.] Likewise, the non-observation of the Higgs boson $H_{2}$ for the same mass value considered, with, this time, the lighter companion of the Higgs pair hiding underneath the experimental bounds for the final-state production rates, leads to a similar formula with $\cos ^{2} \chi$ and $\sin ^{2} \chi$ interchanged and potential cascade corrections supplemented.

When a Higgs boson will be discovered in the present gap down to the LHC improved LEP2 limit, the bound on $\mathcal{R}$ will be replaced by the measured value $\mathcal{R}$ of the ratio of observed to SM cross section, to be explored later. 
Higgs decays into the hidden sector are invisible. This mode however plays quite an important role for hidden-sector scenarios in general. Even though the experimental analysis will be very demanding at hadron colliders [22, 23], less at lepton colliders, the central points should nevertheless be analyzed theoretically in the present context. In a first step, the cross section for $H_{1}$ production with invisible decays may be bounded by

$$
\frac{\sigma\left[p p \rightarrow H_{1} \rightarrow i n v\right]}{\sigma\left[p p \rightarrow H_{1}\right]^{\mathrm{SM}}}=\frac{\sin ^{2} \chi\left[\Gamma_{1}^{\mathrm{hid}} / \Gamma_{\mathrm{tot}, 1}^{\mathrm{SM}}\right]}{1+\tan ^{2} \chi\left[\Gamma_{1}^{\mathrm{hid}} / \Gamma_{\mathrm{tot}, 1}^{\mathrm{SM}}\right]} \leq \mathcal{J}
$$

Analogously, the relation for $H_{2}$ follows immediately by exchanging $\cos ^{2} \chi$ with $\sin ^{2} \chi$ and adding the cascade corrections again. Thus, the total of the visible channels plus the invisible channel describes, globally, the essential elements of the standardhidden Higgs scenario.

Derived from the inequality Eq. 5 above, current experimental exclusion bounds on $\mathcal{R}$ give rise to lower limits on the invisible decay widths in association with the mixing parameters:

$$
\frac{\Gamma_{1}^{\mathrm{hid}}}{\Gamma_{\mathrm{tot}, 1}^{\mathrm{SM}}} \geq \cot ^{2} \chi\left[\frac{\cos ^{2} \chi}{\mathcal{R}}-1\right]
$$

for $\cos ^{2} \chi \geq \mathcal{R}$, while the bound is zero below; similarly for $H_{2}$ by interchanging $\cos ^{2} \chi$ and $\sin ^{2} \chi$ and adding the corrections possibly for cascade decays.

To evaluate the experimental Higgs bounds, we have chosen the characteristic point $\left\{M_{H}=155 \mathrm{GeV} ; \mathcal{R}=0.4\right\}$ within the broad shoulder between $155 \mathrm{GeV}$ and $175 \mathrm{GeV}$, where the observed $\mathcal{R}$ limit is essentially constant [2-4]. Identifying $H$ first with $H_{1}$ and following Eqs. 5, 7, the area above the contour in Fig. 1 is still viable for the potential observation of a Higgs boson with increased luminosity at the LHC. As expected, the exclusion contours move up with reduced $\mathcal{R}$. The corresponding areas for $H$ identified with $H_{2}$ probed for the same mass are just mirrored at $\cos ^{2} \chi=1 / 2$. For $\mathcal{R}<1 / 2$, the $H_{1}$ and $H_{2}$ curves cross each other at a positive value of $\Gamma^{\text {hid }} / \Gamma_{\text {tot }}^{\mathrm{SM}}$, leaving a blank area between the exclusion contours not covered by either Higgs boson.

The ratios $\Gamma^{\text {hid }} / \Gamma_{\text {tot }}^{\mathrm{SM}}$ may run from small up to high values since the Higgs-boson widths in the standard and the hidden sectors may be quite different. The areas dappled by little squares, generated by random points in the space of vacuum expectation values and couplings, is compatible with constraints from unitarity and high-precision measurements, $c f$. Ref. [9]. Small values of $\cos ^{2} \chi$ effectively amount to heavy Higgs scenarios and are disfavored by electroweak precision measurements. The squares nevertheless cover almost the whole allowed parameter space. Note, however, that, when a light $\mathrm{H}_{2}$ is probed, the density of points compatible with the bounds for light $H_{1}$ production [25] is reduced.

The small dot denotes the point where the exclusion bound ends after touching the $\cos ^{2} \chi$-axis at $\mathcal{R}$. For values on the halfaxis $\cos ^{2} \chi$ larger than $\mathcal{R}$ a non-vanishing value of $\Gamma^{\text {hid }}$ is necessary to still be compatible with the experimental bound. In the area below the dotted line the Higgs production cross section [26-30] in association with different numbers of jets [31] is large enough to be accessed experimentally for a luminosity of $50 \mathrm{fb}^{-1}$.

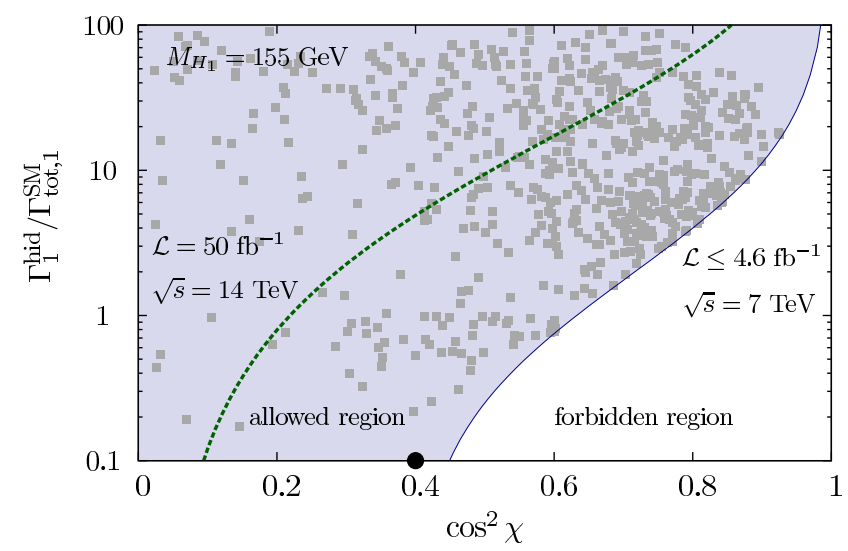

FIG. 1: Bounds on the mixing and hidden decay width of $H_{1}$ for the point $M_{H_{1}}=155 \mathrm{GeV} ; \mathcal{R}=0.4$ in the standard-hidden Higgs scenario, based on current experimental results [2-4]. The regions dappled by small squares are compatible with unitarity and precision measurements. The dot indicates the $\Gamma_{1}^{\text {hid }} \rightarrow 0$ limit of the exclusion curve at $\mathcal{R}$. The dotted line indicates the projected search limit for $\mathcal{L}=50 \mathrm{fb}^{-1}$. The corresponding results for $H_{2}$ with the same mass follow from mirroring all lines at $\cos ^{2} \chi=1 / 2$. 
If an upper bound $\mathcal{J}$ on the invisible cross section can be established, Eq. 6, an upper bound on the Higgs width in the hidden sector follows for $\cos ^{2} \chi \geq \mathcal{J}$ :

$$
\frac{\Gamma_{1}^{\text {hid }}}{\Gamma_{\text {tot }, 1}^{\mathrm{SM}}} \leq \frac{\cot ^{2} \chi \mathcal{J}}{\cos ^{2} \chi-\mathcal{J}}
$$

with, correspondingly, $\cos ^{2} \chi \Leftrightarrow \sin ^{2} \chi$ for $1 \Leftrightarrow 2$ plus cascade contributions. The contour is illustrated in Fig. 2 , with the $68 \%$ $\mathrm{CL}$ region at $50 \mathrm{fb}^{-1}$ luminosity and a center-of-mass energy of $14 \mathrm{TeV}$ given by the shaded area. The shaded area reflects the intrinsic theoretical uncertainties on the perturbative Higgs production cross section [such as pdf and scale uncertainties] as well as the expected experimental uncertainty at the fixed integrated luminosity. All these effects are included in SFitter [32], which we employ to compute the uncertainty band in Fig. 2 [and later analogously in Fig. 3].

In total, $H_{1}$ or $H_{2}$ Higgs bosons with mixing and hidden-width parameters in the allowed regions can, presently, still be realized in the standard-hidden Higgs scenario.

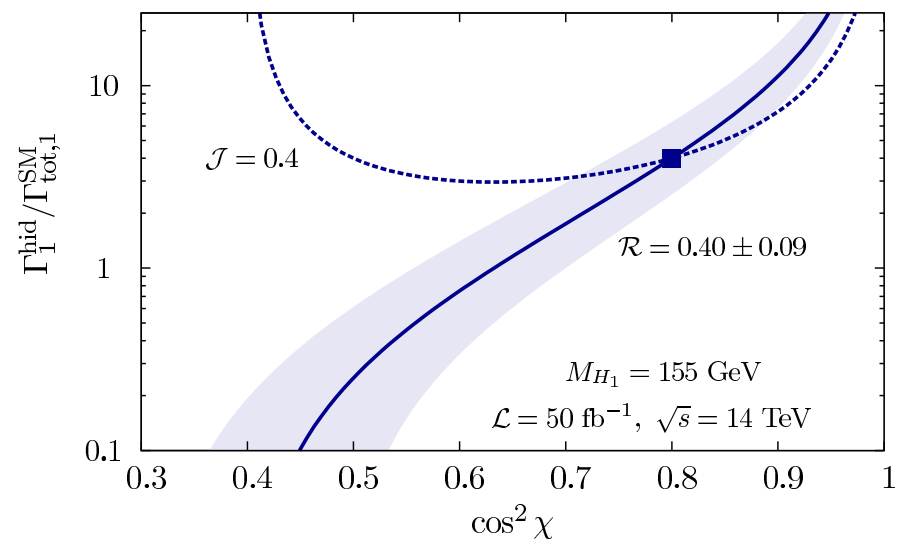

FIG. 2: Contours for mixing and hidden decay width of the Higgs boson $H_{1}$ if visible and invisible ratios of cross sections are measured. The uncertainties of $\mathcal{R}$, shown at 68\% CL as shaded band, are determined by means of SFitter [32] including both experimental and theoretical errors. The uncertainties of $\mathcal{J}$ cannot be reliably estimated at the present time. A currently not excluded Higgs mass of 155 GeV with $\mathcal{R}=\mathcal{J}=0.4$ is chosen for illustration. The crossing point defines the central values of both parameters. The analysis for $H_{2}$ runs parallel after replacing $\cos ^{2} \chi \rightarrow \sin ^{2} \chi$.

\section{Measurements}

Should a light Higgs boson be discovered at the LHC, the measurement of $\mathcal{R}$ provides a first global picture of the physical nature of the state. Instead of just constraining parameter areas, the mixing angle and the width in the hidden sector can then be correlated by exploiting Eq. 7, reduced now to equality. In the limit $\mathcal{R} \rightarrow 1$ the interpretation of the observed state is unique as the $H_{1}$ contour in Fig. 3, for $H_{1}$ identified with the state, shrinks to the SM corner $\left\{\cos ^{2} \chi \rightarrow 1, \Gamma_{1}^{\text {inv }}=\sin ^{2} \chi \Gamma_{1}^{\text {hid }} \rightarrow 0\right\}$. When identifying the observed state with $H_{2}$, the $H_{2}$ contour leads to analogous conclusions in the equivalent limit sin ${ }^{2} \chi \rightarrow 1$. Note that for small mixing the two states $\mathrm{H}_{1}$ and $\mathrm{H}_{2}$, identified consecutively with the state experimentally observed, approach the same Higgs state $H_{s}$ of the Standard Model, as pointed out earlier. Thus the SM Higgs boson emerges in a model-independent way from the experimental data. The agreement with the Standard Model can quantitatively be described by the size of the contour curves approaching the SM corners.

The twin sister of the observed state is difficult to detect experimentally in the SM corner. If the observed state is identified with $H_{1}$, for instance, the production of the higher-mass $H_{2}$ sister is strongly suppressed in the SM approach for an $H_{1}$ mass chosen as $M_{H_{1}}=125 \mathrm{GeV}$ and several $H_{2}$ masses; exemplified for a typical set of parameters:

\begin{tabular}{cc|cc|cc|cc|cc}
\hline $\mathcal{R}_{1}$ & $\mathcal{R}_{2}$ & $M_{H_{1}}[\mathrm{GeV}]$ & $M_{H_{2}}[\mathrm{GeV}]$ & $\cos ^{2} \chi \sin ^{2} \chi$ & $\Gamma_{1}^{\mathrm{hid}} / \Gamma_{1}^{\mathrm{SM}} \Gamma_{2}^{\mathrm{hid}} / \Gamma_{2}^{\mathrm{SM}}$ & $\sigma_{H_{1}}[\mathrm{pb}]$ & $\sigma_{H_{2}}[\mathrm{pb}]$ \\
\hline \hline 0.7 & 0.05 & 125 & 155 & 0.85 & 0.15 & 1.21 & 0.35 & 48.5 & 5.9 \\
& & & 300 & & & & & & 1.9 \\
& & 450 & & & & & & 1.3 \\
\hline
\end{tabular}

Even for a Higgs boson mass of $400 \mathrm{GeV}$, the best combined limit [4] at $\mathcal{R} \sim 0.3$ is not yet sensitive. Thus, the properties of $H_{1}$ must be exploited primarily for studying the nature of the standard-hidden Higgs sector. 
If the invisible parameter $\mathcal{J}$ can be measured experimentally, the crossing of the $\mathcal{J}$ with the $\mathcal{R}$ contour, $c f$. Fig. 2, fixes the two physical parameters at the point marked by the square,

$$
\cos ^{2} \chi=\mathcal{R}_{1}+\mathcal{J}_{1} \quad \text { and } \quad \frac{\Gamma_{1}^{\mathrm{hid}}}{\Gamma_{\text {tot }, 1}^{\mathrm{SM}}}=\frac{\mathcal{J}_{1}}{\mathcal{R}_{1}} \frac{\mathcal{R}_{1}+\mathcal{J}_{1}}{1-\left(\mathcal{R}_{1}+\mathcal{J}_{1}\right)}
$$

for the observed state identified with the Higgs state 1 , and correspondingly $\sin ^{2} \chi$ instead of $\cos ^{2} \chi$ if the observed state is identified with state 2. The result would invite the search for the second Higgs boson of the standard-hidden pair in either case. Depending on the value of $\cos ^{2} \chi$, the wave function of the Higgs boson $H_{1}$, given by $\cos \chi$ and $\sin \chi$, will determine the primary affiliation either with the standard Higgs boson $H_{s}$ or the hidden Higgs boson $H_{h}$, and complementary for $H_{2}$. Note, however, that the nearly parallel running of the $\mathcal{R}$ and $\mathcal{J}$ curves for large $\cos ^{2} \chi$ in Fig. 2 renders the measurement of the crossing point experimentally very difficult when the errors on $\mathcal{J}$ are included.

We conclude by observing that the two Higgs masses $M_{1,2}$ and the ratios $\mathcal{R}_{1,2}$ and $\mathcal{J}_{1,2}$ measured for $H_{1,2}$ complete, in general, the parameter determination of this theory, i.e. the elements of the Higgs matrix and the hidden widths of the Higgs field $H_{s}$ at the respective physical Higgs mass values. In fact, the measurement of all the ratios $\mathcal{R}_{1,2}$ and $\mathcal{J}_{1,2}$ over-determines the parameter set, and the sum rule $\mathcal{R}_{1}+\mathcal{J}_{1}+\mathcal{R}_{2}+\mathcal{J}_{2}=1$ can be exploited to test the consistency of the standard-hidden Higgs scenario with the experimental results.

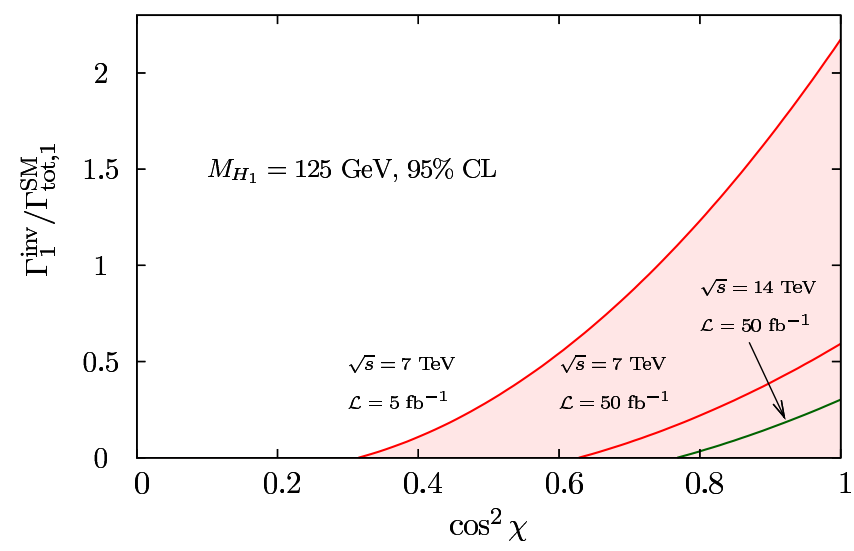

FIG. 3: 95\% CL contours for the mixing and hidden decay width from the observation of a SM-like Higgs boson [R $=1]$ of mass 125 GeV. The uncertainty bands for different LHC energies and luminosities are determined by means of SFitter [32] including both experimental and theoretical errors. To focus on the SM limit proper, $\left\{\cos ^{2} \chi, \Gamma_{1}^{i n v}\right\} \rightarrow\{1,0\}$, the observable $\Gamma_{1}^{i n v}$ is chosen as parameter in the figure.

\section{CONCLUSIONS}

Expanding on earlier theoretical analyses, we have exploited recent LHC data to draw conclusions on the Higgs sector in a standard-hidden Higgs scenario which develops continuously out of the Standard Model. Coupling the Standard Model with a hidden sector suggests an exciting interpretation of the recent experimental data.

- Mixing effects and invisible decays of Higgs particles can reduce the cross sections for experimental searches of the Higgs boson at the LHC. Though the SM Higgs boson may be ruled out for specific mass values, if cross sections fall below the SM predictions, Higgs bosons are not excluded per se for these mass values. Constraints then follow for the mixing of standard and hidden Higgs states, as well as for decays into the hidden sector. Such Higgs bosons should emerge however with rising LHC luminosity.

- If a Higgs boson is discovered at the LHC, the standard-hidden Higgs scenario allows to quantify how closely the state coincides with or to what extent it deviates from the Standard Model. It is demonstrated in Fig. 3 how from present collider parameters such a measurement will rapidly gain sensitivity with increasing luminosity and energy.

Thus, the standard-hidden Higgs system not only describes an exciting physical scenario, but it can also be adopted for interpreting the data in searches for Higgs bosons at the LHC and, if discovered, for the global understanding of their nature. 


\section{Acknowledgments}

C. Englert and P. M. Zerwas are grateful to Heidelberg University for the warm hospitality extended to them during several visits. C. Englert acknowledges funding by the Durham International Junior Research Fellowship scheme. M. Rauch acknowledges support by the Deutsche Forschungsgemeinschaft via the Sonderforschungsbereich/Transregio SFB/TR-9 "Computational Particle Physics" and the Initiative and Networking Fund of the Helmholtz Association, contract HA-101 ("Physics at the Terascale").

[1] F. Englert and R. Brout, Phys. Rev. Lett. 13 (1964) 321; P. W. Higgs, Phys. Lett. 12 (1964) 132 and Phys. Rev. Lett. 13 (1964) 508; G. S. Guralnik, C. R. Hagen and T. W. B. Kibble, Phys. Rev. Lett. 13 (1964) 585.

[2] ATLAS Collaboration, G. Aad et al., Eur. Phys. J. C 71, 1728 (2011).

[3] CMS Collaboration, S. Chatrchyan et al., Phys. Lett. B699 (2011) 25.

[4] ATLAS and CMS collaborations, ATLAS-CONF-2011-157, CMS PAS HIG-11-023.

[5] ATLAS Collaboration, arXiv:1112.2577 [hep-ex]; ATLAS-CONF-2011-161; ATLAS-CONF-2011-162; ATLAS-CONF-2011-163; all available under http: / / cdsweb.cern.ch/collection/ATLAS 20 Conference 20 Notes

[6] CMS Collaboration, CMS-PAS-HIG-11-025, CMS-PAS-HIG-11-029, CMS-PAS-HIG-11-030, CMS-PAS-HIG-11-031, CMS-PAS-HIG11-032;

all available under http://cdsweb.cern.ch/collection/CMS20Physics 20 Analysis 20 Summaries

[7] R. Barate et al., Phys. Lett. B 565 (2003) 61.

[8] S. Bock, R. Lafaye, T. Plehn, M. Rauch, D. Zerwas and P. M. Zerwas, Phys. Lett. B 694 (2010) 44; see also Ref. [10].

[9] C. Englert, T. Plehn, D. Zerwas and P. M. Zerwas, Phys. Lett. B 703 (2011) 298.

[10] J. R. Espinosa, C. Grojean and M. Mühlleitner, JHEP 1005, 065 (2010).

[11] for early work see e.g. T. Binoth and J. J. van der Bij, Z. Phys. C 75, 17 (1997); B. Patt and F. Wilczek, arXiv:hep-ph/0605188.

[12] V. Barger, P. Langacker, M. McCaskey, M. J. Ramsey-Musolf and G. Shaughnessy, Phys. Rev. D 77, 035005 (2008); E. E. Boos, S. V. Demidov and D. S. Gorbunov, Int. J. Mod. Phys. A 26, 3201 (2011); K. Ghosh, B. Mukhopadhyaya and U. Sarkar, Phys. Rev. D 84, 015017 (2011); S. V. Demidov, D. S. Gorbunov and A. A. Tokareva, arXiv:1111.1072 [hep-ph]; J. F. Kamenik and C. Smith, arXiv:1111.6402 [hep-ph].

[13] M. J. Strassler and K. M. Zurek, Phys. Lett. B 651, 374 (2007); M. J. Strassler and K. M. Zurek, Phys. Lett. B 661, 263 (2008).

[14] L. B. Okun, Sov. Phys. JETP 56 (1982) 502 [Zh. Eksp. Teor. Fiz. 83 (1982) 892]; B. Holdom, Phys. Lett. B 166, 196 (1986); N. ArkaniHamed and N. Weiner, JHEP 0812, 104 (2008); M. Ahlers, J. Jaeckel, J. Redondo and A. Ringwald, Phys. Rev. D 78, 075005 (2008); J. Jaeckel and A. Ringwald, Ann. Rev. Nucl. Part. Sci. 60 (2010) 405; E. Weihs and J. Zurita, arXiv:1110.5909 [hep-ph].

[15] S. Dodelson and L. M. Widrow, Phys. Rev. Lett. 72 (1994) 17; J. March-Russell, S. M. West, D. Cumberbatch and D. Hooper, JHEP 0807, 058 (2008).

[16] T. Plehn, arXiv:0910.4182 [hep-ph], to appear as Springer Lecture Notes in Physics 844.

[17] for some recent updates see e.g. M. Shaposhnikov and C. Wetterich, Phys. Lett. B 683, 196 (2010); M. Holthausen, K. S. Lim, and M. Lindner [arXiv: 1112.2415 [hep-ph]].

[18] D. E. Morrissey, T. Plehn and T. M. P. Tait, arXiv:0912.3259 [hep-ph].

[19] R. Schabinger and J. D. Wells, Phys. Rev. D 72, 093007 (2005); M. Bowen, Y. Cui and J. D. Wells, JHEP 0703, 036 (2007); J. D. Wells, In *Kane, Gordon (ed.), Pierce, Aaron (ed.): Perspectives on LHC physics* 283-298 [arXiv:0803.1243 [hep-ph]].

[20] A. Djouadi, P. Janot, J. Kalinowski and P. M. Zerwas, Phys. Lett. B 376 (1996) 220 [arXiv:hep-ph/9603368].

[21] R. E. Shrock and M. Suzuki, Phys. Lett. B 110 (1982) 250; K. Belotsky, D. Fargion, M. Khlopov, R. Konoplich and K. Shibaev, Phys. Rev. D 68 (2003) 054027; E. Ma, arXiv:1109.4177 [hep-ph] and arXiv:1112.1367 [hep-ph].

[22] see e.g. O. J. P. Eboli, D. Zeppenfeld, Phys. Lett. B495, 147-154 (2000).

[23] R. Foot, A. Kobakhidze and R. R. Volkas, arXiv:1109.0919 [hep-ph]; C. Englert, J. Jaeckel, E. Re and M. Spannowsky, arXiv:1111.1719 [hep-ph]; Y. Bai, J. Fan and J. L. Hewett, arXiv:1112.1964 [hep-ph]; B. A. Dobrescu, G. D. Kribs and A. Martin, arXiv:1112.2208 [hep-ph].

[24] J. McDonald, Phys. Rev. D 50, 3637 (1994); C. P. Burgess, M. Pospelov and T. ter Veldhuis, Nucl. Phys. B 619, 709 (2001); R. Barbieri, T. Gregoire and L. J. Hall, hep-ph/0509242; M. H. G. Tytgat, PoSIDM 2010, 126 (2011); O. Lebedev and H. M. Lee, arXiv:1105.2284 [hep-ph]; M. Pospelov and A. Ritz, arXiv:1109.4872 [hep-ph]; X. -G. He and J. Tandean, Phys. Rev. D 84, 075018 (2011); P. J. Fox, R. Harnik, J. Kopp and Y. Tsai, arXiv:1109.4398 [hep-ph]; I. Low, P. Schwaller, G. Shaughnessy and C. E. M. Wagner, arXiv: 1110.4405 [hep-ph]; J. -W. Cui, H. -J. He, L. -C. Lv and F. -R. Yin, arXiv:1110.6893 [hep-ph]; O. Lebedev, H. M. Lee and Y. Mambrini, arXiv:1111.4482 [hep-ph]; Y. Mambrini, arXiv:1108.0671 [hep-ph] and arXiv:1112.0011 [hep-ph]; S. Baek, P. Ko and W. -I. Park, arXiv:1112.1847 [hep-ph]. B. Batell, S. Gori and L. -T. Wang, arXiv:1112.5180 [hep-ph].

[25] LEP Higgs Working Group, hep-ex/0107032.

[26] H. M. Georgi, S. L. Glashow, M. E. Machacek and D. V. Nanopoulos, Phys. Rev. Lett. 40 (1978) 692D; A. Djouadi, M. Spira and P. M. Zerwas, Phys. Lett. B 264 (1991) 440; S. Dawson, Nucl. Phys. B 359, 283 (1991); M. Spira, A. Djouadi, D. Graudenz and P. M. Zerwas, Nucl. Phys. B 453 (1995) 17; R. V. Harlander and W. B. Kilgore, Phys. Rev. Lett. 88, 201801 (2002); S. Moch and A. Vogt, Phys. Lett. B 631, 48 (2005).

[27] D. L. Rainwater, D. Zeppenfeld and K. Hagiwara, Phys. Rev. D 59, 014037 (1998); N. Kauer, T. Plehn, D. L. Rainwater and D. Zeppenfeld, Phys. Lett. B 503, 113 (2001).

[28] S. L. Glashow, D. V. Nanopoulos and A. Yildiz, Phys. Rev. D 18, 1724 (1978); J. Finjord, G. Girardi and P. Sorba, Phys. Lett. B 89, 
99 (1979); E. Eichten, I. Hinchliffe, K. D. Lane and C. Quigg, Rev. Mod. Phys. 56 (1984) 579 [Addendum-ibid. 58 (1986) 1065];

B. A. Kniehl and C. P. Palisoc, arXiv:1112.1575 [hep-ph].

[29] W. Beenakker, S. Dittmaier, M. Krämer, B. Plümper, M. Spira and P. M. Zerwas, Phys. Rev. Lett. 87 (2001) 201805; S. Dawson, C. Jackson, L. H. Orr, L. Reina and D. Wackeroth, Phys. Rev. D 68 (2003) 034022.

[30] S. Catani, D. de Florian, M. Grazzini and P. Nason, JHEP 0307, 028 (2003); K. Arnold et al., Comput. Phys. Commun. 180 (2009) 1661.

[31] E. Gerwick, T. Plehn and S. Schumann, Phys. Rev. Lett. in print, arXiv:1108.3335 [hep-ph].

[32] R. Lafaye, T. Plehn, M. Rauch, D. Zerwas and M. Dührssen, JHEP 0908, 009 (2009); M. Rauch, arXiv:1110.1196 [hep-ph]. 